\title{
Advanced Laparoscopic surgery: Our initial experience at Nepal Medical College
}

\author{
Nishes, Subash, Pranil, Prof SKS
}

Department of Surgery, Nepal Medical College Teaching Hospital, Atterkhel, Kathmandu

Correspondence: Asso. Prof Dr Pranil, Lecturer, Department of Surgery, Nepal Medical College Teaching Hospital

\begin{abstract}
If we consider the last century of history of surgery, few advances can be compared to the changes engendered by the introduction of minimally invasive surgery, which in the last decade has represented a revolution in surgical practice and patient care. Since 1987, when the first laparoscopic cholecystectomy was performed, laparoscopic procedures have been the standard of care for many routine diagnostic and therapeutic procedures. Laparoscopic techniques have become an integral part of the operative management in virtually every realm of general surgery. The success of the laparoscopic technique has been due mainly to patient demand, which has contributed to a rapid expansion in the number of laparoscopic procedures performed
\end{abstract}

The benefits conferred to patients by less invasive procedures like decreased pain, and shorter recovery time have to be weighed against overzealous application and the problems created by the lack of familiarity with new techniques and instruments. Basic laparoscopic procedures include laparoscopic cholecystectomy, laparoscopic appendectomy, and diagnostic laparoscopy. All other laparoscopic operations are defined as "advanced". Learning curve and added expenses have been prohibitative in development of advanced laparoscopic surgery in resource poor setting like ours. However, advance laparoscopic surgery is here to stay. We must therefore have a concentrated effort to move towards offering these facilities to our patients.

Despite the initial hiccups, some advanced laparoscopic surgeries are being performed and at Nepal Medical College, moving a step closer towards the gold standards in surgical management. Surgeons from various units and subspecialties work together and help each other and utilize each other's limited experience. This spirit has been a great help to reach our way to develop ourselves despite our technical limitations. Our experience is limited but the outcomes so far are encouraging and we have tried to highlight the significance of framework. There is a long road ahead but a hood beginning is more than half done.

Key words: Laproscopic, Nephrectomy, Fundoplication, TEP, Appendectomy 